\title{
EXISTENCE OF SOLUTIONS AND SEMI-DISCRETIZATION FOR PDE WITH INFINITE DELAY
}

\section{PIRIADARSHANi AND T. SENGADIR}

Abstract. In this paper, we obtain an existence theorem for a Semi-Linear PDE with infinite delay employing a phase space in which discretizations can naturally be performed. Further, for linear PDEs with infinite delay we show that the solutions of the ODE with infinite delay obtained by the semi-discretization converge to the original solution. Our results cover various types of PDEs under the assumption that semi-discretization of the PDEs without the delay terms can be performed. The method of our proof is applicable for the case of finite delays too.

Mathematics subject classification (2010): 35-XX, 35R10, 35A01.

Keywords and phrases: semi-linear PDE, approximation, semi-discretization, infinite delay.

\section{REFERENCES}

[1] M. Adimy, H. BouZAhIR AND K. EZZINBI, Existence for a class of partial functional differential equations with infrite delay, Nonlinear Analysis, 46 (2001), 91-112.

[2] D. Agirseven, Approximate solutions of delay parabolic equations with the dirichlet condition, Abstract and Applied Analysis, Article ID 682752 (2012).

[3] J. Dyson, R. Villella-Bressan And G.F. Webba, Semilinear transport equation with delays, IJMMS, 32 (2003), 2011-2026.

[4] J.K. Hale And J. Kato, Phase space for retarded equations with infinite delay, Funkcialaj Ekvacioj, 21, 1 (1978), 11-41.

[5] C. Huang And S. Vandewalle, Unconditionally stable difference methods for delay partial differential equations, Numerische Mathematik, 122 (2012), 579-603.

[6] K. ITO AND F. KAPPEL, The Trotter-Kato theorem and approximation of PDE's, Math. Of Comp, 67, 221 (1998), 21-44.

[7] Z. JACKIEWICZ AND B. ZUBIK-KowAL, Spectral collocation and waveform relaxation methods for nonlinear delay partial differential equations, Appl. Numer. Math., 56 (2006), 433-443.

[8] N.P. Kopystyra AND A.T. SAKhTAGANOVA, Convergence of the method of lines for one quasilinear parabolic equation with delay, Journal of Mathematical Sciences, 63 (1993), 522-527.

[9] S.H. KUlKarni And G. RAMEsh, Projection methods for inversion of unbounded operators, Indian J. Pure and Appl. Math., 39 (2008), 185-202.

[10] J. LiAnG AND T. XIAO, Functional differential equations with infinite delay in Banach spaces, Internat. J. Math. and Math. Sci, 14 (1991), 497-508.

[11] D.J. MARTN Higham AND T. SARDAR, Existence and stability of fixed points for a discretised nonlinear reaction-diffusion equation with delay, Appl. Numer. Math., 18 (1995), 155-173.

[12] J. Mead And B. ZubiK-Kowal, An iterated Pseudo spectral method for delay partial differential equations, Appl. Numer. Math., 55 (2005), 227-250.

[13] A. PAZY, Semigroups of linear operators and applications to partial differential equations, Springer Verlag, New York, 1983.

[14] M. Roales, F. Rodrguez And J.A. Martin, Exact solutions and numerical approximations of mixed problems for the wave equation with delay, Applied Matheamtics and Computation, 219 (2012), 3178-3186. 
[15] A.D. ReY AND M.C. MACKEY, Multi-stability and boundary layer development in a transport equation with delayed arguments, Canad. Appl. Math. Quart, 1 (1993), 61-81.

[16] T. Sengadir, Discretisation of an infinite delay equation, Math. Comput., 76 (2006), 777-793.

[17] P.J. Van Der Houwen, B.P. Sommeijer And C.T.H. BaKer, On the stability of predictorcorrector methods for parabolic equations with delay, IMA J. Numer. Anal, 6 (1986), 1-23.

[18] J. VAN LENT, J. JANSSEN AND S. VANDEWALle, Multigrid waveform relaxation for delay partial differential equations, Manuscript.

[19] B. ZUBIK-KOWAL AND S. VANDEWALLE, Waveform relaxion for functional differential equations, SIAM J. Sci. Comput, 21 (1999), 207-226.

[20] B. ZubiK-KowaL, Chebyshev Pseudo spectral method and waveform relaxation for differential and differential-functional parabolic equations, Appl. Numer. Math., 34 (2000), 309-328.

[21] B. ZUBIK-Kowal, Error bounds for spatial discretization and waveform relaxation applied to parabolic functional differential equations, J. Math. Anal. Appl, 293 (2004), 496-510. 